\title{
DINÂMICA QUANTITATIVA POPULACIONAL: UMA SIMULAÇÃO *
}

\author{
(Recebido em 21/7/1969)
}

\author{
E. P. dos SANTos
}

Departamento de Biologia Geral da Faculdade de Filosofia, Ciências e Letras da Universidade de São Paulo e Instituto Oceanográfico da Universidade de São Paulo

\section{SYNOPSIS}

This paper presents a method of investigating the dynamics of a biological population, using a technique of simulation with a digital electronic computer.

Its purpose is to show an analysis of a simulated exploited population.

\section{INTRODUÇÃO}

A Análise Populacional tem por objetivo a determinação da "estrutura" e da "dinâmica" das populações biológicas. Procura determinar, num dado instante, a quantidade (estrutura quantitativa) de indivíduos existente na população ou em classes da população, a maneira como êsses indivíduos se dis. tribuem na região em que vivem (estrutura espacial) e como variam, em função do tempo, o número (di. nâmica quantitativa) e a distribuição (dinâmica espacial) dêsses indivíduos. Procura determinar, também, a existência de interações entre as populações.

Denominamos "classes de uma população" a conjuntos de indivíduos, com determinadas características comuns, existentes nessa população.

A metodologia tradicional empregada em estudos populacionais consiste de análises quantitativas com modêlos matemáticos "analíticos". Infelizmente, o tratamento analítico de sistemas biológicos complexos (WATt, 1962) é difícil e muitas vêzes impossível.

O principal objetivo dêste trabalho é apresentar uma técnica de simulação com computadores eletrônicos digitais, para o estudo da "dinâmica quantitativa", evitando o tratamento analítico.

Utilizamos, para a elaboração dessa técnica, os computadores IBM 360/44 do Setor de Matemática Aplicada "Prof. Dr. Walter Schutzer" e o IBM 1620 do Centro de Computação Eletrônica do Instituto de Pesquisas Matemáticas, ambos da Universidade de São Paulo.

Vários pesquisadores estrangeiros têm trabalhado em "dinâmica quantitativa populacional", porém uti-

* Parte da tese de livre-docência apresentada à Cadeira de Biologia Geral, Faculdade de Filosofia, Ciências e Letras da Universidade de São Paulo. lizando modêlos matemáticos analíticos. Atualmente, alguns dêsses pesquisadores (G. J. Paulik, S. C. King, C. J. Pennycuick e D. Garfinkel) estão começando a estudar sistemas simulados, porém determinísticos. Este trabalho apresenta uma simulação estocástica (com variáveis aleatórias).

Um dos primeiros modêlos analíticos elaborados, porém utilizado até hoje na investigação pesqueira (Beverton \& Holt, 1957), tem as seguintes premissas:

1) $\operatorname{NVS}(\mathrm{t}+\Delta \mathrm{t})-\mathrm{NVS}(\mathrm{t})=\mathrm{NR}-\mathrm{NM}$

2) $\mathrm{NR}=\mathrm{R} \cdot \mathrm{NVS}(\mathrm{t}) \cdot \Delta \mathrm{t}$

3) $\mathrm{NM}=\mathrm{M} \cdot \mathrm{NVS}(\mathrm{t}) \cdot \Delta \mathrm{t}$

onde: $\operatorname{NVS}(\mathrm{t})$ e NVS $(\mathrm{t}+\Delta \mathrm{t})=$ tamanho da população nos instantes $\mathrm{t}$ e $\mathrm{t}+\Delta \mathrm{t}$,

$\mathrm{NR}=$ número de indivíduos que entra para a população, por nascimento ou migração, no intervalo de tempo $\Delta \mathrm{t}$.

$\mathrm{NM}=$ número de indivíduos que sai da população, por morte ou migração, no intervalo de tem. po $\Delta t$.

$$
\begin{gathered}
\Delta \lim _{\mathrm{t} \rightarrow 0} \frac{\operatorname{NVS}(t+\Delta t)-\operatorname{NVS}(t)}{\Delta t}=\frac{d \operatorname{NVS}(t)}{d t}= \\
=(R-M) \cdot \operatorname{NVS}(t) \\
\int \frac{d \operatorname{NVS}(t)}{\operatorname{NVS}(t)}=(R-M) \int d t
\end{gathered}
$$

conseqüência :

$$
\operatorname{NVS}(t)=\mathrm{e}^{(\mathrm{R}-\mathrm{M})(\mathrm{t}+\mathrm{c})}
$$


A premissa (2) é válida se a capacidade ambiental fôr ilimitada.

Se a capacidade ambiental fôr limitada $(K)$ à medida que NVS $(t)$ tende para $K$, NR pode diminuir, segundo a expressão:

$$
\mathrm{NR}=\mathrm{R} \cdot \mathrm{NVS}(\mathrm{t}) \cdot \Delta \mathrm{t} \cdot\left(1-\frac{\mathrm{NVS}(\mathrm{t})}{\mathrm{K}}\right)
$$

portanto

$$
\begin{aligned}
\frac{\mathrm{d} \operatorname{NVS}(\mathrm{t})}{\mathrm{dt}} & =(\mathrm{R}-\mathrm{M}) \cdot \operatorname{NVS}(\mathrm{t})- \\
- & \frac{\mathrm{R}}{\mathrm{K}} \cdot \operatorname{NVS}(\mathrm{t})^{2}
\end{aligned}
$$

conseqüência :

$$
\operatorname{NVS}(t)=\frac{R-M}{K+e^{(R-M)}} \frac{(t+c)}{(t+2)}
$$

Leslie (1948) imaginou uma população em que a fecundidade e a taxa de mortalidade eram constantes, porém diferentes para as diversas classes etárias.

Fazendo:

$\mathrm{VV}_{\mathrm{i}, \mathrm{t}}=$ número de fêmeas da classe etária i, no tempo t,

$\mathrm{F}_{\mathrm{i}}=$ número médio de descendentes por fêmea da classe etária $i$, por unidade de tempo, e

$\mathrm{P}_{\mathrm{i}} \quad=$ probabilidade de sobrevivência de cada fêmea da classe etária i, por unidade de tempo,

podemos escrever para $\Delta \mathrm{t}=1$ (unidade de tempo):

$$
\begin{aligned}
& \sum_{\mathrm{i}=1}^{\mathrm{n}} \mathrm{F}_{\mathrm{i}} \cdot \mathrm{NV}_{\mathrm{i}, \overline{0}}^{-}=\mathrm{NV}_{0,1} \\
& \mathrm{P}_{0} \cdot \mathrm{NV}_{0,0}=\mathrm{NV}_{1,1} \\
& \mathrm{P}_{1} \cdot \mathrm{NV}_{1,0}=\mathrm{NV}_{2,1} \\
& \mathrm{P}_{\mathrm{n}-1} \cdot \mathrm{NV}_{\mathrm{n}-1,0}=\mathrm{NV}_{\mathrm{n}, 1}
\end{aligned}
$$

representando matricialmente:

$$
\left[\begin{array}{cccc}
\mathrm{F}_{1} & \mathrm{~F}_{2} & \ldots & \mathrm{F}_{\mathrm{n}} \\
\mathrm{P}_{0} & & & \\
& \mathrm{P}_{1} & & \\
& & & \\
& & \mathrm{P}_{\mathrm{n}-1}
\end{array}\right] \times\left[\begin{array}{c}
\mathrm{NV}_{0,0} \\
\mathrm{NV}_{1,0} \\
\cdot \\
\cdot \\
\mathrm{NV}_{\mathrm{n}-1,0}
\end{array}\right]=\left[\begin{array}{c}
\mathrm{NV}_{0,1} \\
\mathrm{NV}_{1,1} \\
\cdot \\
\cdot \\
\mathrm{NV}_{\mathrm{n}-1,1}
\end{array}\right]
$$

Se fizermos $F_{i}$ e $P_{i}$ variarem em função do tamanho da população, a manipulação da expressão matricial acima torna-se difícil.
Pennycuick et al. (1968) imaginou u'a maneira de resolver o problema "simuladamente". Após cada produto das matrizes, uma nova matriz de $F_{i}$ e $\mathrm{P}_{\mathrm{i}}$ era determinada, em função do tamanho da população. O procedimento foi processado com computadores eletrônicos digitais.

Silliman (1967) apresenta uma simulação analógica dêste modêlo. KING \& PAULIK (1967) substituiram a simulação analógica, por uma digital.

Concluindo, os estudos populacionais simulados estão ainda em fase inicial.

\section{MÉTODO}

\section{A) O MODÊLO}

Em uma população biológica, as seguintes premissas (Prem.) podem ser válidas:

Prem. 1 - A variação, num certo intervalo de tempo, no número de indivíduos adultos de uma população depende apenas do número de indivíduos jovens, que se tornam adultos (número de recrutas), e do número de adultos que morre, nesse intervalo de tempo.

Nota: Dividimos a população em duas classes, a de jovens (imaturos) e a de adultos.

Prem. $2-\mathrm{O}$ número de recrutas, num certo intervalo de tempo, depende apenas do tamanho da população e da capacidade am. biental (para jovens), nesse intervalo de tempo.

\section{Definições:}

"Capacidade ambiental" é o número máximo de indivíduos de uma população, ou de uma determinada classe de uma população, que pode sobreviver em uma região, devido à quantidade disponível de espaço e de alimento e às condições físico-químicas do meio ambiente. Este valor independe, portanto, da mortalidade devida à doenças e predadores (inclusive o homem).

"Potencial biológico" é o quociente entre o número de indivíduos que uma população. ou uma determinada classe de uma população, poderia ter se o ambiente fôsse ilimitado no momento da reprodurão ou do recrutamento, e o número que realmente existe.

Prem. 3 - $\mathrm{O}$ número de adultos que morre, num certo intervalo de tempo, depende apenas do tamanho da população e da intensidade da exploração humana (esfôrço), nesse intervalo de tempo.

Nota: Nêste modêlo, não nos preocupamos com a sobrevivência devida às condições ambientais para os adultos e à longevidade.

Infelizmente, a maioria dos trabalhos em biologia populacional não apresenta explìcitamente as pre- 
missas envolvidas. Essa disposição das informações facilita o raciocínio e a exposição.

\section{B) A Simulação}

Nêste trabalho, um dos principais propósitos da simulação é produzir variação ao acaso nos parâmetros envolvidos (Modêlo Simulado Estocástico): Todo o estudo poderia ser feito, em princípio, utilizando técnicas de sorteio. Entretanto, devido à quantidade de operações envolvidas, isto seria humanamente im. possivel. Felizmente, podemos gerar números ao acaso com computadores eletrônicos digitais (Kuo, 1965). Esses computadores substituem, portanto, a técnica de sorteio, executando ràpidamente as operações.

\section{1) A natalidade}

Segundo a Prem. 2, supondo a capacidade am. biental infinita, podemos escrever:

onde:

$$
\mathrm{NN}=\mathrm{PN} . \mathrm{NVS}
$$

$\mathrm{NN}=$ número de jovens que "podem" tornar-se adultos.

NVS = tamanho da população,

$\mathrm{PN}=$ coeficiente de proporcionalidade.

Mas sendo $\mathrm{NJ}$ a capacidade ambiental real para os jovens, se $\mathrm{NN} \leq \mathrm{NJ}$, teremos

$$
\mathrm{NN}=\text { número de recrutas, }
$$

se $\mathrm{NN}>\mathrm{NJ}$,

NN deverá sofrer uma taxa de mortalidade igual a

$$
\mathrm{TJ}=\frac{\mathrm{NN}-\mathrm{NJ}}{\mathrm{NN}} \quad 0 \leq \mathrm{TJ} \leq 1
$$

TJ mede a probabilidade de morte de cada indivíduo dos $\mathrm{NN}$.

Se gerarmos NN números ao acaso (YFL), no intervalo $(0,1)$, o que pode ser feito usando a seguinte sub-rotina em linguagem Fortran IV, para computador IBM $360 / 44$ :

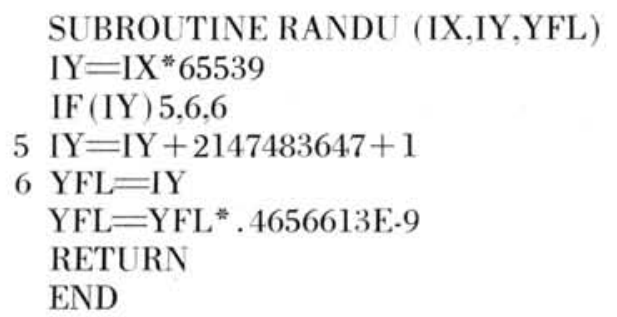

e determinarmos o número $(\mathrm{NM})$ de $\mathrm{YFL} \leq \mathrm{TJ}, \mathrm{NM}$ será uma variável aleatória, com distribuição binomial (Tocher, 1963) e representará o número de jovens mortos devido às condições ambientais.

Portanto $\mathrm{NN}-\mathrm{NM}=$ número de recrutas.
Estas operações foram representadas no diagra. ma de bloco da Figura 1.

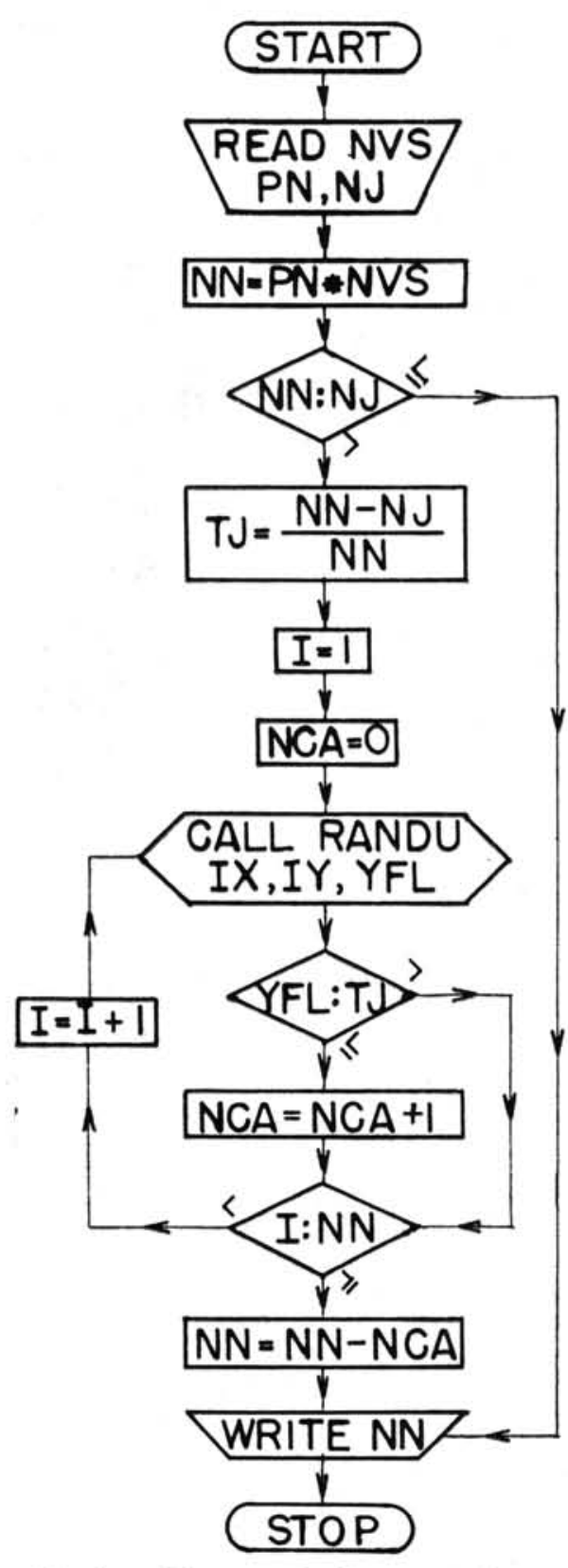

Fig. 1 - Diagrama de bloco que simula a Prem. 2, onde: NVS = tamanho da populacão no inlcio de um intervalo de tempo; $\mathrm{NJ}=$ capacidade ambiental para os jovens; $\mathrm{NN}=$ número de recrutas; $\mathrm{PN}=$ proporcão entre NN e NVS, para $\mathrm{NJ}=\infty ; \mathrm{YFL}=$ número ao acaso no intervalo (0 1), gerado pela sub-rotina RẢNDU apresentada no texto.

\section{2) A mortalidade natural}

Segundo a Prem. 3, na ausência de exploração humana, $\mathrm{TN}=$ taxa de mortalidade natural (exceto exploração humana). 


$$
0 \leq \mathrm{TN} \leq 1
$$

TN mede a probabilidade de morte (devida à causas naturais) de cada indivíduo adulto da população (NVS).

Se gerarmos NVS números ao acaso (YFL), no intervalo $(0,1)$, o que pode ser feito com a sub-rotina RANDU citada, e determinarmos o número (NM) de $\mathrm{YFL} \leq \mathrm{TN}$, êsse número será uma variável aleatória com distribuição binomial (Tocher, 1963), e representará o número de adultos que morre, devido a causas naturais (exceto o homem).

Estas operações foram representadas no diagrama de bloco da Figura 2a.
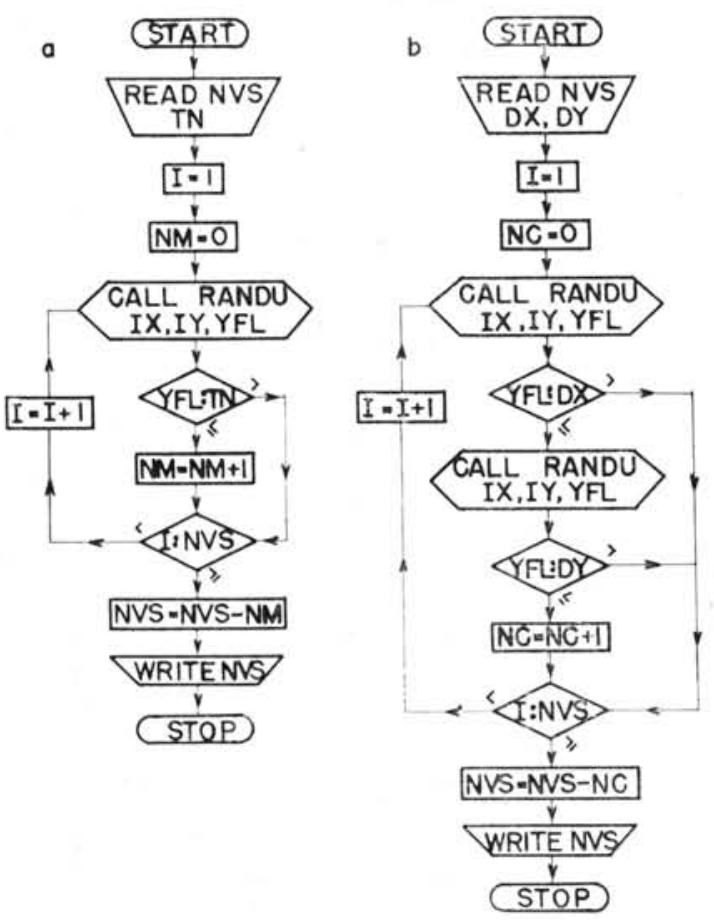

Fig. 2 - Diagrama de bloco que simula NVS individuos - a: sob taxa TN de mortalidade natural e b: sob uma única captura fixa na posição I da Fig. 3a, de dimensões DX e DY, na ausência de mortalidade natural. YFL $=$ números ao acaso no intervalo $(0,1)$, gerado pela sub-rotina RANDU apresentada no texto.

\section{3) A exploração humana}

Podemos (SANTos, 1968a e b) representar os indivíduos de uma população, distribuidos em uma região bi ou tridimensional, através de pontos distribuidos em um gráfico (Fig. 3a).

As capturas dêsses indivíduos, efetuadas pelo homem, com rêdes, coletores, armadilhas, etc., em locais dispostos ao acaso na região, equivalem à retirada dos indivíduos de determinadas áreas (ou volumes) dispostas ao acaso na região.

Se lançarmos retângulos ao acaso na Figura 3a e determinarmos o número (NC) de pontos abran-
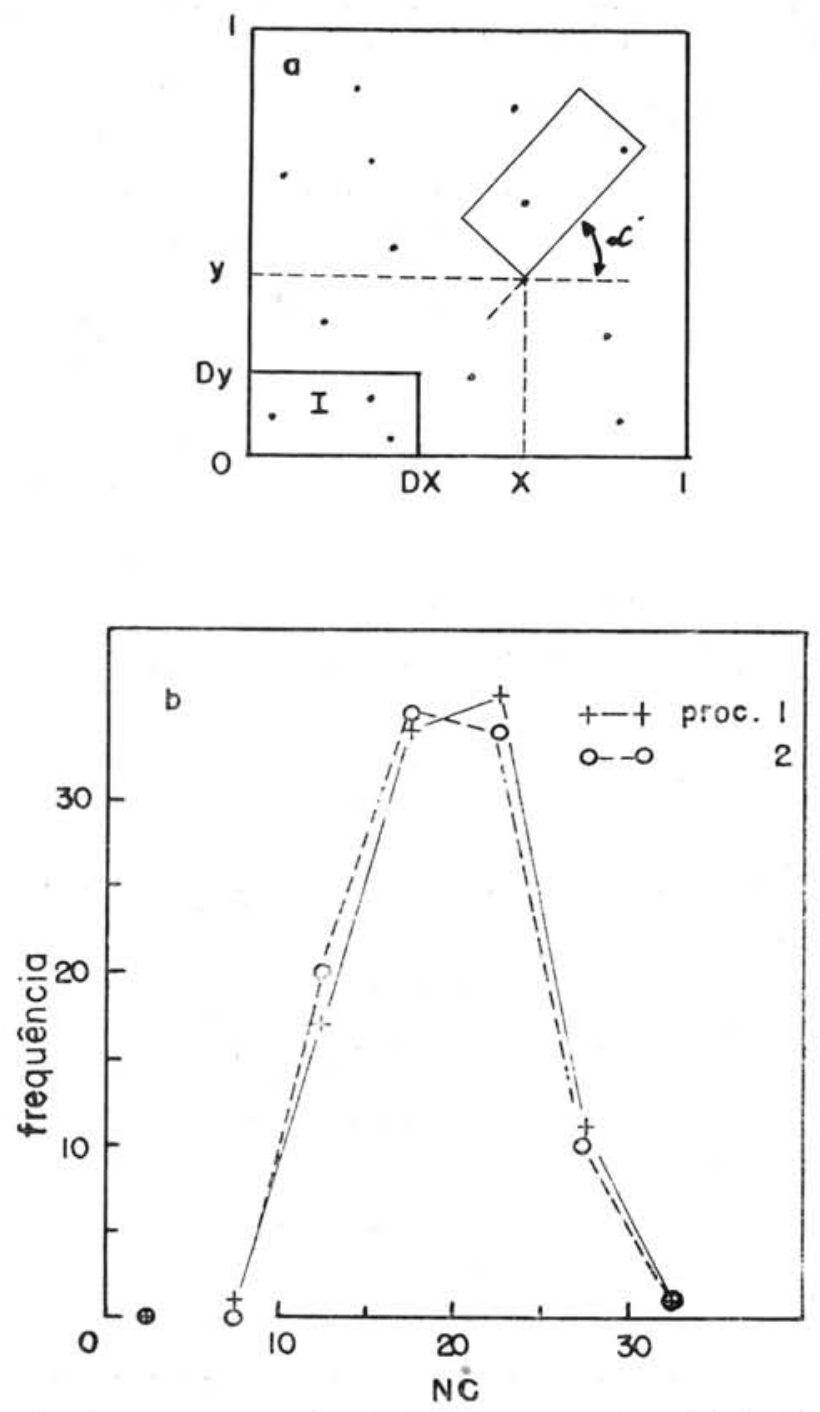

Fig. 3 - a: Representação de uma população distribuıda em uma região bidimensional e de algumas cap. turas ao acaso; b: distribuições de frequência de NC obtidas através dos dois procedimentos (proc.) apresentados no texto.

gidos por êsses retângulos, êsse procedimento simula capturas em uma população. NC representa o número de indivíduos capturados.

Um retângulo terá posição ao acaso em um gráfico (Fig. 3a), se as coordenadas (X,Y) de um de seus vértices forem números ao acaso, no intervalo $(0,1)$ em nosso caso, e se a reta, que contiver um de seus lados, tiver declive (oc) ao acaso, no intervalo $\left(0^{\circ}, 360^{\circ}\right)$

Se DX e DY forem as dimensões do retângulo, é necessário que as coordenadas do vértice estejam no intervalo:

$$
\left(\sqrt{\mathrm{DX}^{2}+\mathrm{DY}^{2}}, 1-\sqrt{\left.\mathrm{DX}^{2}+\mathrm{DY}^{2}\right)}\right.
$$

segundo o teorema de Pitágoras, a fim de que o retângulo não caia, nem em parte, fora do gráfico. 
O diagrama de bloco da Figura 4 representa as seguintes operações:

I) Gera NN pontos ao acaso em um gráfico quadrado (Fig. 3a) com dimensões $1 \times 1$.

II) Gera NQ retângulos com dimensões DX e DY dispostos ao acaso no gráfico.

III) Determina o número $\mathrm{NC}$ de pontos abrangidos por retângulo.

Um procedimento (Proc. 2) equivalente a êste e que simplifica as operações mencionadas, consiste em gerar NQ conjuntos de NN pontos ao acaso e determinar o número $\mathrm{NC}$ de pontos de cada conjunto. abrangido pelo retângulo na posição I da Figura 3a. Isto se os pontos tiverem distribuição ao acaso na região. As coordenadas dos pontos devem ser números ao acaso no intervalo $(0,1)$.

$\mathrm{O}$ diagrama de bloco da Figura $2 \mathrm{~b}$ representa as operações envolvidas neste segundo procedimento.

A Figura 3b apresenta as distribuiçôes de freqüência de $\mathrm{NC}$, obtidas com os dois procedimentos. Segundo o teste de aderência de Kolmogorov-Smirnov $(1-K(0,4)=0,997>0,05)$ não há diferença significativa entre as duas distribuições e portanto entre os dois procedimentos.

\section{4) As interações}

Sejam:

NVS $=$ tamanho (em número de indivíduos) de uma população, no início de um certo intervalo de tempo,

$\mathrm{TN}=$ taxa de mortalidade natural, no intervalo de tempo, e

$\mathrm{NQ}=$ número de capturas (iguais) efetuadas nês. se intervalo de tempo.
Surge assim o seguinte problema: "Qual a taxa de mortalidade total da população, nêsse intervalo de tempo?" Isto é, qual a interação existente entre a mortalidade natural e a devida à exploração humana?

A fim de resolver êsse problema, imaginamos o seguinte procedimento:

I) Inicialmente, dividimos o intervalo de tem. po em NH sub-intervalos.

Segundo Ricker (1958):

$$
\mathrm{TN}=1-\mathrm{e}^{-\mathrm{m}_{\mathrm{N}}}
$$

onde: $\mathrm{m}_{\mathrm{N}}=$ coeficiente instantâneo de mortalidade natural, no intervalo de tempo.

O coeficiente instantâneo de mortalidade natural em um sub-intervalo será:

$$
\frac{\mathrm{m}_{\mathrm{N}}}{\mathrm{NH}}
$$

Portanto a taxa de mortalidade natural no subintervalo será:

$$
1-\mathrm{e}^{-\frac{\mathrm{mi}_{\mathrm{N}}}{\mathrm{NH}}}
$$

II) Em seguida, geramos NQ números ao acaso no intervalo $(1, \mathrm{NH})$. Êstes números indicam os sub-intervalos de tempo, em que houve capturas.

III) Nos sub-intervalos sem captura, a população ficou sujeita apenas à mortalidade natural (Fig. 2a). Nos sub-intervalos com captura, a população ficou sujeita inicialmente à mortalidade imposta pela captura (Fig. 2b). Depois os indivíduos remanes. centes ficaram sujeitos à mortalidade natural (Fig. 2a).

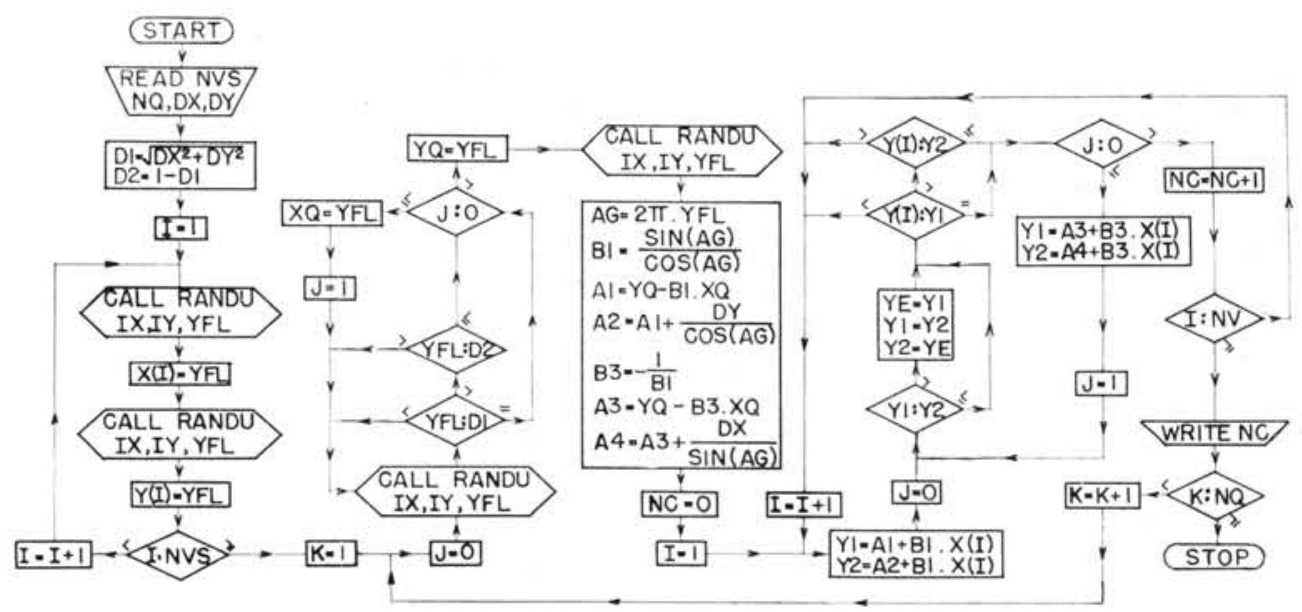

Fig. 4 - Diagrama de bloco que simula NQ capturas ao acaso, com dimensōes DX e DY, em uma populacão com NVS individuos. YFL $=$ número ao acaso no intervalo $(0,1)$, gerado pela sub-rotina RANDU apresentada no texto; $N C=$ número de individuos capturados. 
A Figura 5 apresenta a distribuição de freqüên. cia do coeficiente instantâneo de mortalidade total, medido em 30 populações com 100 indivíduos cada, e com:

$$
\begin{aligned}
& \mathrm{TN}=0,3 \\
& \mathrm{NQ}=10 \\
& \mathrm{NH}=20
\end{aligned}
$$

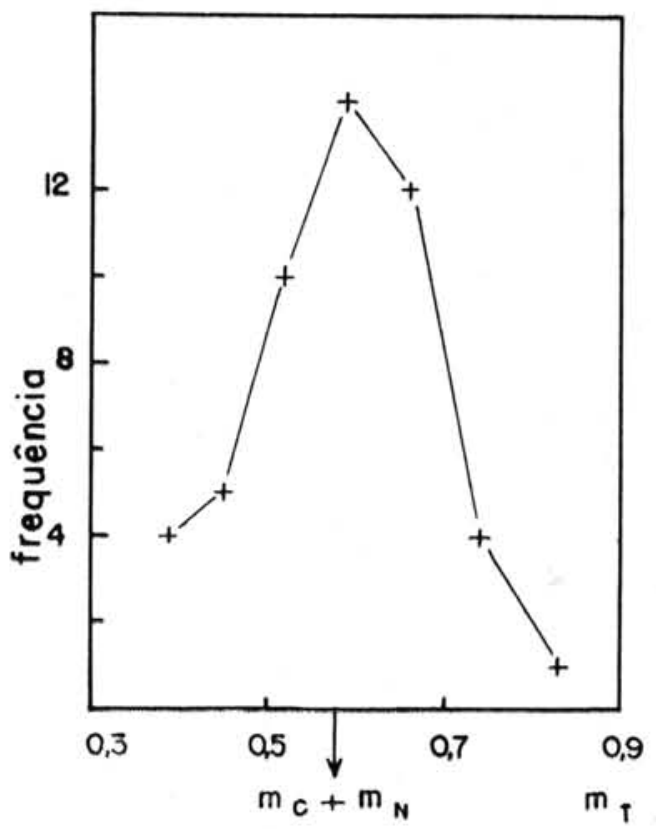

Fig. 5 - Distribuição de freqüência do coeficiente instantâneo de mortalídade total $\left(m_{\mathrm{T}}\right)$.

Sendo $\mathrm{DX}=0,2$ e DY $=0,1$, as dimensões de cada captura, na ausência de mortalidade natural, a esperança matemática do número de indivíduos capturado $(\mathrm{E}(\mathrm{NC}))$ será 20 , o que corresponde a um coeficiente instantâneo de mortalidade devida à captura igual a 0,223 .

Sendo $\mathrm{TN}=0,3$, a esperança matemática do coeficiente instantâneo de mortalidade natural será de 0,357 .

Podemos notar que o valor médio da distribuição apresentada na Figura 5 é igual à soma das esperan. ças matemáticas citadas. Esta afirmação é confirmada pelo teste de hipótese de que a média de uma distri. buição tem um valor especificado, quando o desvio padrão é desconhecido $(\mathrm{p}(\mathrm{t})>0,05)$.

Podemos escrever:

$$
\mathrm{m}_{\mathrm{T}}=\mathrm{m}_{\mathrm{N}}+\mathrm{m}_{\mathrm{c}}
$$

onde: $\mathrm{m}_{\mathrm{T}}=$ coeficiente instantâneo de mortalidade total,

$\mathrm{m}_{\mathrm{N}}=$ coeficiente instantâneo de mortalidade natural, e

$\mathrm{m}_{\mathrm{c}}=$ coeficiente instantâneo de mortalidade devida à captura. (1958).

Esste resultado está de acôrdo com RicKER

\section{C) As Consequências}

Reunindo as informações apresentadas, resulta o seguinte programa em linguagem Fortran IV:

C DIQUA - E. P. DOS SANTOS

DIMENSION NV(100), NC(100), NR(100)

READ $(5,1)$ NG,NJ,L,NH,NQ,IX

READ $(5,2)$ TN,PN,DX,DY

$\mathrm{ANH}=\mathrm{NH}$

$\mathrm{PN}=\mathrm{PN} / \mathrm{ANH}$

$\mathrm{CN}=\operatorname{ALOG}(1 .-\mathrm{TN})$

$\mathrm{CN}=\mathrm{CN} / \mathrm{ANH}$

$\mathrm{TN}=1 .-\mathrm{EXP}(\mathrm{CN})$

$\operatorname{READ}(5,1)(\mathrm{NV}(\mathrm{I}), \mathrm{I}=1, \mathrm{~L})$

DO 3 IN $=1, N G$

$\mathrm{NN}=0$

DO $16 \mathrm{I}=1, \mathrm{~L}$

$16 \mathrm{NC}(\mathrm{I})=0$

DO $4 \mathrm{I}=1, \mathrm{NQ}$

CALL RANDU (IX,IY,W

$\mathrm{IX}=\mathrm{IY}$

$4 \mathrm{NR}(\mathrm{I})=\mathrm{W}^{*} \mathrm{ANH}$

DO $17 \mathrm{I}=1, \mathrm{NH}$

DO $6 \mathrm{~J}=1, \mathrm{NQ}$

$15 \mathrm{IF}(\mathrm{I}-\mathrm{NR}(\mathrm{J})) 6,7,6$

6 CONTINUE

GO TO 8

7 DO $9 \mathrm{~K}=1, \mathrm{~L}$

$\mathrm{NVF}=\mathrm{NV}(\mathrm{K})$

IF (NVF) $9,9,10$

$10 \mathrm{NCF}=0$

DO $11 \mathrm{M}=1, \mathrm{NVF}$

CALL RANDU (IX,IY,X)

$\mathrm{IX}=\mathrm{IY}$

IF (X-DX) $12,12,11$

12 CALL RANDU(IX,IY,Y)

$\mathrm{IX}=\mathrm{IY}$

IF (Y - DY) $13,13,11$

$13 \mathrm{NCF}=\mathrm{NCF}+1$

11 CONTINUE

$\mathrm{NV}(\mathrm{K})=\mathrm{NVF}-\mathrm{NCF}$

$\mathrm{NC}(\mathrm{K})=\mathrm{NC}(\mathrm{K})+\mathrm{NCF}$

9 CONTINUE

IF ( J - NQ ) 14,8,8

$14 \mathrm{~J}=\mathrm{J}+1$

GO TO 15

8 DO $17 \mathrm{~K}=1, \mathrm{~L}$

$\mathrm{NVF}=\mathrm{NV}(\mathrm{K})$

IF (NVF) $17,17,18$

$18 \mathrm{NM}=0$

DO $19 \mathrm{M}=1, \mathrm{NVF}$

CALL RANDU (IX,IY,RN)

$\mathrm{IX}=\mathrm{IY}$

IF (RN-TN) $20,20,19$

$20 \mathrm{NM}=\mathrm{NM}+1$

19 CONTINUE

$\mathrm{FNV}=\mathrm{NVF}-\mathrm{NM}$

$\mathrm{NVP}=\mathrm{PN}^{*} \mathrm{FNV}$

$\mathrm{NN}=\mathrm{NN}+\mathrm{NVP}$

$\mathrm{NV}(\mathrm{K})=\mathrm{FNV}$

17 CONTINUE

$\mathrm{M}=\mathrm{L}$

DO $21 \mathrm{I}=1, \mathrm{M}$ 


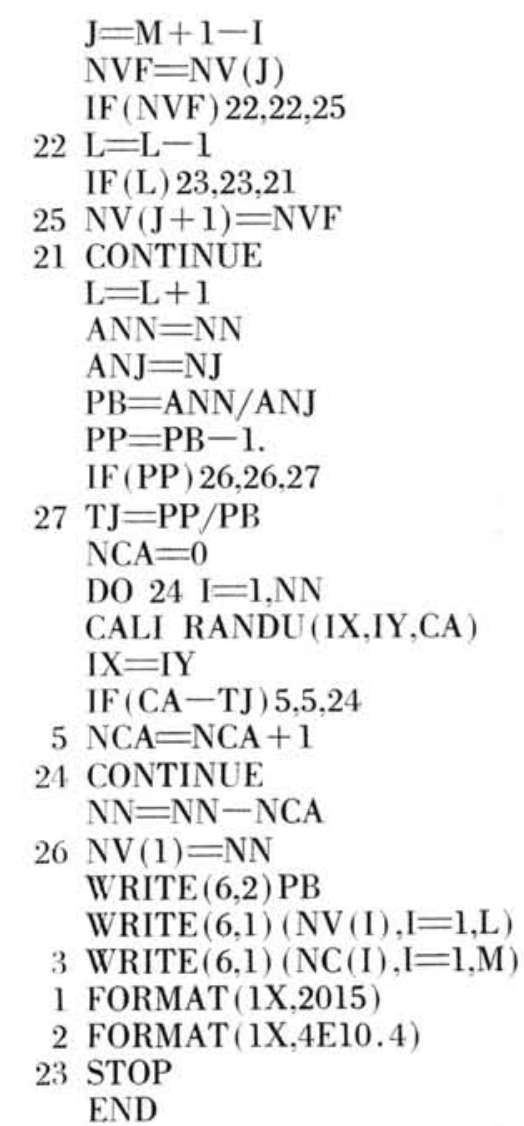

onde: entrada -

NG $=$ número de intervalos de tempo,

NJ $\quad=$ capacidade ambiental para os jovens,

L $\quad=$ número de classes etárias (longevida. de),

$\mathrm{NH}=$ nmero de sub-intervalos,

NQ $=$ número de capturas,

IX = número inteiro ímpar,

TN $=$ taxa de mortalidade natural por intervalo de tempo,

PN = proporção entre o tamanho da popula. ção (NVS) e o número de jovens que poderiam tornar-se adultos, se NJ fôs. se infinito,

DX e DY $=$ dimensões das capturas,

$\mathrm{NV}(\mathrm{I})=$ número de indivíduos da classe $\mathrm{I}$ de idade, existente na população,

saída -

$\mathrm{PB}=$ potencial biológico,

$\mathrm{NV}(\mathrm{I})=$ número de indivíduos da classe $\mathrm{I}$ de idade, existente na população,

$\mathrm{NC}(\mathrm{I})=$ número de indivíduos capturado da classe I de idade.

Este programa simula uma população com uma determinada natalidade, mortalidade natural e devida à captura.

\section{1." Conseqüência}

Com o programa DIQUA, fazendo:

$$
\begin{array}{lll}
\mathrm{TN}=0,3 & \mathrm{NH}=20 & \mathrm{DX}=0,2 \\
\mathrm{NJ}=100 & \mathrm{PN}=0,5 & \mathrm{DY}=0,1
\end{array}
$$

e variando NQ, obtivemos NV(I) em diferentes intervalos de tempo (NG).

Calculamos a taxa de mortalidade total (TM(I)), por intervalo de tempo, para a classe I de idade, com a expressão:

$$
\mathrm{TM}(\mathrm{I})=\frac{\mathrm{NV}(\mathrm{I}, \mathrm{J})-\mathrm{NV}(\mathrm{I}, \mathrm{J}+\mathrm{I})}{\mathrm{NV}(\mathrm{I}, \mathrm{J})}
$$

onde: $\quad \mathrm{NV}(\mathrm{I}, \mathrm{J})=$ número de indivíduos da classe I de idade, no instante J,

$\mathrm{NV}(\mathrm{I} . \mathrm{J}+\mathrm{I})=$ idem, no instante $\mathrm{J}+\mathrm{l}$,

e o coeficiente instantâneo de mortalidade total $\left(\mathrm{m}_{\mathrm{T}(1)}\right)$ com a expressão:

$$
\mathrm{TM}(\mathrm{I})=1-\mathrm{e}^{-\mathrm{m}_{\mathrm{T}}(\mathrm{I})}
$$

Na Figura 6 lançamos $\mathrm{m}_{\mathrm{T}(1)}$ médio $\left(\mathrm{m}_{\mathrm{T}}\right)$, para $\mathrm{NV}(\mathrm{I}, \mathrm{J})>10$ indivíduos e o mesmo esfôrço (NQ) para $\mathrm{NG}=10$, em função de $\mathrm{NQ}$.

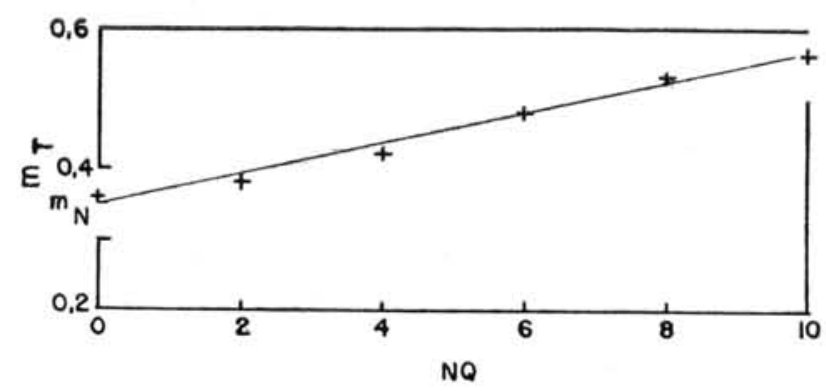

Fig. 6 - Dependência entre o coeficiente instantâneo do mortalidade total médio $\left(\mathrm{m}_{\mathrm{T}}\right)$ € o esfôrço (NQ). $\mathrm{m}_{\mathrm{N}}=$ coeficiente instantâneo de mortalidade natural.

Podemos notar que a dependência é linear e:

$$
\mathrm{m}_{\mathrm{T}}=\mathrm{m}_{\mathrm{N}} \quad \text { para } \quad \mathrm{NQ}=0
$$

Concluindo, a primeira conseqüência simulada, obtida do modêlo, é:

"A dependência entre o coeficiente instan. tâneo médio de mortalidade total e o esfôrço, em uma população explorada pelo homem, é linear, com o têrmo independente igual ao coeficiente instantâneo de mortalidade natural, suposto cons. tante".

Esta dependência tem sido observada em várias populações (SANTos et al., 1969). 


\section{2." Conseqüência}

A Figura 7 apresenta a variação no potencial biológico $(\mathrm{Pb})$, no número $(\mathrm{NN})$ de recrutas e no tamanho (NVS) de uma população simulada com o programa DIQUA.

Os dados utilizados são os mesmos da $1 .^{\text {a }}$ Conseqüência, porém $\mathrm{NQ}=0,10$ e 20 .

Inicialmente $(\mathrm{NQ}=0)$, a população encontra. -se em equilíbrio. Os parâmetros mencionados per. manecem constantes:

$$
\begin{aligned}
& \mathrm{Pb} \cong 2 \\
& \mathrm{NVS} \cong 300 \text { indivíduos } \\
& \mathrm{NN} \cong 100 \text { indivíduos }
\end{aligned}
$$

Aumentando o esfôrço $(\mathrm{NQ}=10)$, NVS e $\mathrm{Pb}$ começam a diminuir (fase de pré-equilíbrio), entrando em seguida em equilíbrio, novamente:

$$
\begin{aligned}
& \mathrm{Pb} \cong 1,25 \\
& \mathrm{NVS} \cong 240 \text { individuos } \\
& \mathrm{NN} \cong 100 \text { indivíduos }
\end{aligned}
$$

$\mathrm{O}$ número de recrutas não variou.

Aumentando novamente o esforç̧o $(\mathrm{NQ}=20)$, NVS e $\mathrm{Pb}$ diminuem até zero. $\mathrm{O}$ número de recrutas permanece constante enquanto $\mathrm{Pb}>1$. diminuin. do para $\mathrm{Pb}<1$ (fase de depredação).
Para $1>\mathrm{Pb}>0.5$, se eliminarmos o esfôrço $(\mathrm{NQ}=0)$, os parâmetros voltam à situação inicial (fase de regeneração).

Para $\mathrm{Pb}<0,5$, mesmo eliminando o esfôrço, os parâmetros continuam a diminuir até zero (fase de auto-depredação).

$$
\begin{aligned}
& \mathrm{Pb}=1 \quad \text { nível crítico } \\
& \mathrm{Pb}=0,5 \text { (em nosso caso) nível super-crítico }
\end{aligned}
$$

Concluindo, de acôrdo com o modêlo proposto. uma população poderá ser explorada continuamente enquanto $\mathrm{Pb}>$ nível crítico. $\mathrm{O}$ aumento do esfôrço (intensidade de exploração) acarreta diminuição no tamanho da população. Entretanto, o equilíbrio será estabelecido, se o esfôrço fôr constante.

Para:

$$
\text { nível super-crítico }<\mathrm{Pb}<\text { nível crítico, }
$$

a exploração deve ser intermitente, com períodos sem exploração, para que haja a regeneração da população.

Para:

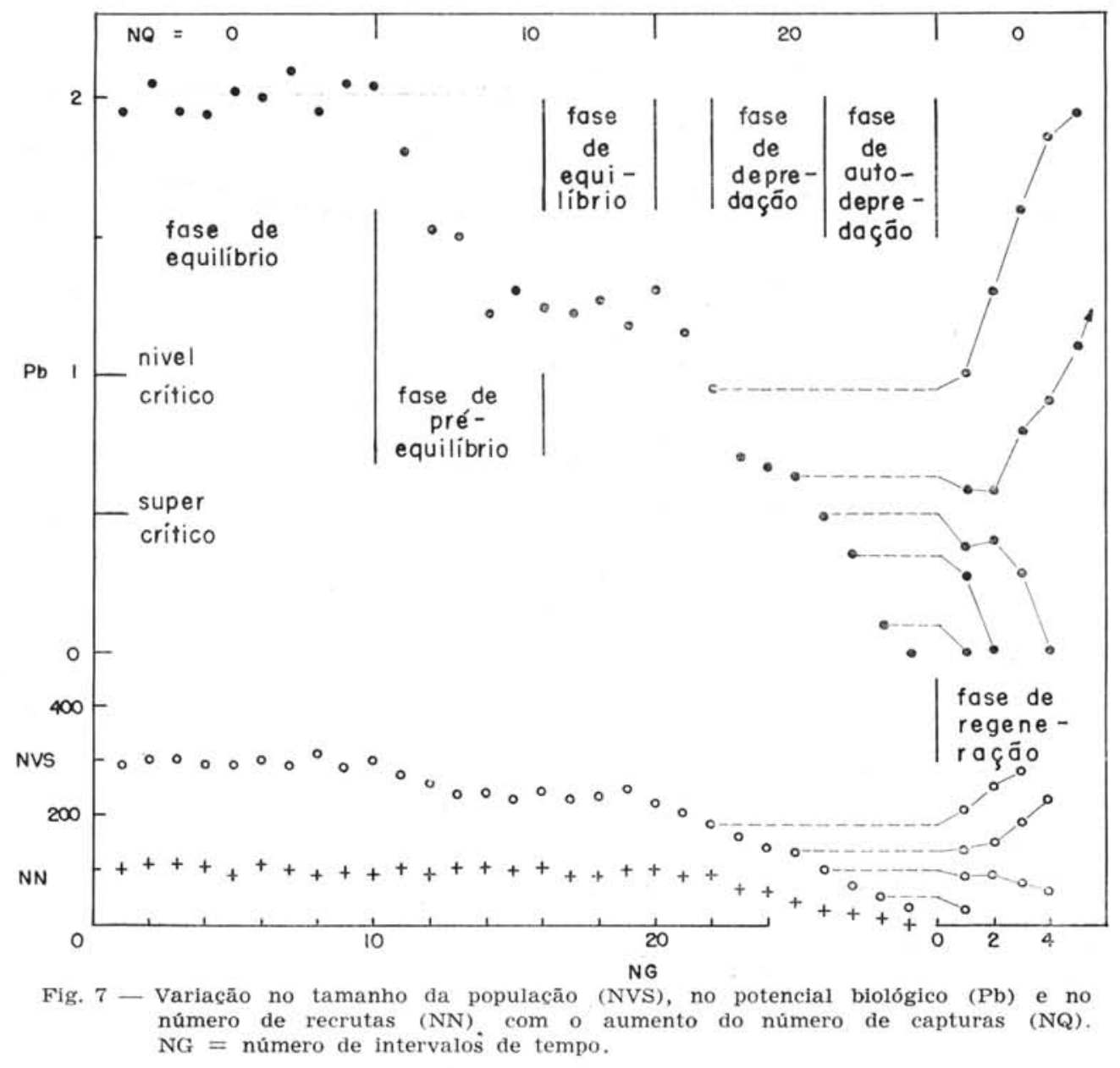


mesmo eliminando o esfôrço, a população desaparecerá.

"Nêste caso $\mathrm{NQ}=20$ é denominado esfôrço predatório".

\section{CONCLUSOES}

Se as premissas do modêlo apresentado forem válidas em uma população, o aumento da taxa de mortalidade diminui $o$ tamanho da população $\left(2 .^{\mathrm{t}}\right.$ conseqüência).

$\mathrm{Na}$ investigação pesqueira, o têrmo "depredação" tem sido usado, quando o tamanho da população simplesmente diminui, sob a ação da pesca, ou quando a população desaparece. Segundo o modêlo apresentado, haverá diminuição no tamanho da população, sempre que o esfôrço fôr aumentado. Isto sugere, que o têrmo depredação deve ser usado quando a intensidade de exploração (esfôrço predatório) põe em perigo a existência da população $(\mathrm{Pb}<1)$.

\section{AGRADECIMENTOS}

Somos profundamente grato ao Prof. Dr. A. B. da Cunha, ao Prof. Dr. C. Pavan, à Dra. M. Vannucci, ao Centro de Computação Eletrônica da USP, ao Setor de Matemática Aplicada "Prof. Dr. Walter Schutzer" da USP e às Srtas. M. Garido e T. Picard.

\section{B I B L I O G R A F I A}

Beverton, R. J. H. \& Holt, S. J.

1957. On the dynamics of exploited fish populations. Fish. Invest., Lond., ser. 2, vol. $19,535 \mathrm{p}$.

KING, C. S. \& PaUliK, G. J.

1967.

Dynamic models and the simulation of ecological systems. J. theor. Biol., vol. 16 , n. 2 , p. $251-267$.
Kuo, S. S.

1965.

Numerical methods and computers. Massachusetts, Addison-Wesley, $341 \mathrm{p}$.

LESLIE, P. H.

1948. Some further notes on the use of matrices in population mathematics. Biometrika, vol. 35 , p. $213-245$

Pennycuick, C. J., Compton, R. M.

\& RECKINGHAM, L.

1968. A computer model for simulating the growth of a population, or two interacting populations. J. theor. Biol., vol. 18, n. 3, p. 316-329.

RICKER, W. E.

1958. Handbook of computations for biological statistics of fish populations. Bull. Fish. Res. Bd Can., n. 119,300 p.

Santos, E. P. dos

1968a. Distribuições populacionais. Bolm Inst. oceanogr., vol. 17, p. 1-15.

1968b. Populational distribution I: the digital simulation. Mar. biol., vol. 1, n. ${ }^{\circ} 4$ p. $348-350$.

Santos, E. P. dos, Neiva, G. S. \& Shaeffer, Y.

1969. Dinâmica da população de camarão sete-barbas, Xiphopeneus kroyeri (Heller) na baía de Santos. Bolm SUDEPE, n. 2. (No prelo.)

Silliman, R. P.

1967.

Analog computer models of fish population. Fishery Bull. Fish Wildl. Serv. U.S., vol. 66 , n. ${ }^{\circ} 1$, p. $31-46$

TOCHER, K. D.

1963. The art of simulation. London, English Univ. Press, 148 p.

WatT, K. E. F.

1962. Use of mathematics in population ecology. A. Rev. Ent., vol. 7, p. 243-260. 